\title{
Cavity ringdown spectroscopy for the analysis of small liquid volumes
}

\author{
Claire Vallance and Cathy M. Rushworth
}

Cavity-enhanced absorption techniques show considerable promise for applications in which a high detection sensitivity is required while maintaining a small probed volume of liquid sample. Conventional two-mirror cavity ringdown or cavityenhanced absorption spectroscopy appears to provide a simple general 'benchtop' platform for measurements on flow cells and microfluidic chips with suitable optical properties, and recent progress in developing integrated cavities on-chip hints at the potential for future miniaturised self-contained microfluidic devices.

\section{Introduction}

When developing chemical sensors, minimising the sample volume required for a successful test is often a key goal. In some situations this may be because only very limited amounts of sample are available, for example in forensics and some medical tests, while in others there may be a desire to increase the portability of the sensor, to reduce the costs associated with expensive reagents, or to minimise the amount of chemical waste generated. In the medical arena, reduced sample volume generally reduces the invasiveness of the test, for example replacing a venous blood draw with a fingerprick blood test, or allows an increased number of tests to be carried out on a given sample volume.

One of the limiting factors in reducing the sample volume is the availability of detection techniques with sufficient sensitivity to detect extremely small quantities of the chemical species of interest. A variety of such techniques are available, each

Claire Vallance

Department of Chemistry, University of Oxford, Chemistry Research Laboratory, 12 Mansfield Rd, Oxford OX1 3TA, UK, e-mail: claire.vallance@chem.ox.ac.uk

Cathy M. Rushworth

Department of Chemistry, University of Oxford, Chemistry Research Laboratory, 12 Mansfield Rd, Oxford OX1 3TA, UK, e-mail: catherine.rushworth@chem.ox.ac.uk 
with their own strengths and weaknesses. In the field of microfluidics, for example, where sample volumes tend to lie in the microlitre to picolitre range, detection methods include electrochemical techniques [1], fluorescence [2] and Raman spectroscopy [3, 4], nuclear magnetic resonance spectroscopy [5], and mass spectrometry [6], amongst others $[7,8,9,10,11,12,13,14,15]$. To date, absorption spectrosocopy has been fairly conspicuous by its absence in microfluidic applications. While having considerable appeal as a detection method for chemical sensing, since every chemical species absorbs light in some region of the electromagnetic spectrum and may therefore be detected assuming suitable light sources and detectors are available, difficulties arise when dealing with very small sample volumes. This is easily illustrated by considering the well-known Beer-Lambert law, which describes the fraction of light transmitted through a sample as a function of the analyte concentration, $C$, the path length through the sample, $l$, and the absorption coefficient $\alpha$ or extinction coefficient $\varepsilon$ of the sample molecule, which quantify the efficiency with which the molecule absorbs light.

$$
\frac{I}{I_{0}}=e^{-\alpha C l}=10^{-\varepsilon C l}
$$

Here, $I_{0}$ and $I$ denote the light intensity incident on and transmitted through the sample. In general laboratory applications of absorption spectroscopy, the liquid sample is contained within a cuvette of path length $\sim 1 \mathrm{~cm}$. Reducing the sample volume to microfluidic dimensions or similar often yields a path length so short that the absorption becomes undetectable. Inspection of Equation (1) reveals that the detection sensitivity can be improved by increasing the absorption/extinction coefficient, the sample concentration, or the path length through the sample. Since the absorption coefficient is a constant of the molecule under study ${ }^{1}$, and the concentration is generally a constant of the sample, increasing the path length through the sample is often the only option. As we shall see, cavity-enhanced spectroscopic techniques have the potential to achieve significant increases in absorption path length, and therefore detection sensitivity, without increasing the sample volume, and show considerable promise as a means of amplifying the absorption signal in small-volume liquid samples.

When dealing with gas-phase samples, we have seen from other chapters that cavity-enhanced spectroscopic methods provide a huge improvement in detection sensitivity over single-pass absorption methods. The minimum detectable absorption per unit path length, $\kappa=\alpha C$, is typically in the range from $10^{-6}$ to $10^{-9} \mathrm{~cm}^{-1}$ [16], with detection limits as low as $8 \times 10^{-11}$ having been achieved using some of the more sophisticated approaches [17]. As a result, cavity-enhanced approaches have become the spectroscopic detection methods of choice for applications ranging from fundamental studies of molecular spectroscopy, kinetics and dynamics, through to atmospheric and environmental sensing, and air and process quality monitoring $[18,16,19,20,21]$. There has also been considerable recent interest in the

\footnotetext{
${ }^{1}$ See Section 5 for a discussion of colourimetric cavity ringdown spectroscopy, which uses colourimetric reactions to convert the molecule of interest into a strongly absorbing derivative compounds, thereby effectively increasing $\alpha$.
} 
potential for developing medical diagnostic and monitoring devices based on the absorption signatures of biomarkers present in trace amounts within exhaled breath $[22,23,24,25,26]$. In the liquid phase, the much higher density of molecules means that there are fewer situations in which the level of signal amplification offered by a cavity-enhanced absorption technique is required. Also, for a variety of reasons (explored in Section 3), the sensitivity enhancements achievable for liquid-phase samples are generally less than for gaseous samples. These two facts taken together have meant that applications of cavity-enhanced spectroscopies to measurements on liquid-phase samples are much less common than in the gas phase, with most reports to date comprising proof-of-concept studies on possible cavity configurations $[27,28,29,30,31,32,33,34,35,36]$. However, as noted above, there is considerable scope for using such techniques when probing small-volume liquid samples.

We begin this chapter with a brief introduction to the two most commonlyused cavity-enhanced absorption techniques, namely cavity ringdown spectroscopy (CRDS) and cavity-enhanced absorption spectroscopy (CEAS), focusing on the simplest experimental configuration, in which the cavity is formed from a pair of carefully-aligned high-reflectivity plano-concave mirrors. We then outline the challenges associated with incorporating liquid samples into an optical cavity and summarise the various approaches that have been adopted. Finally, we explore a number of potential application areas in microfluidics and chemical sensing.

\section{Fundamentals of cavity ringdown and cavity-enhanced absorption spectroscopy}

\subsection{General principles of cavity ringdown spectroscopy}

A typical experimental setup for a cavity ringdown or cavity-enhanced absorption spectroscopy measurement is shown schematically in Figure 1. The basis of the cavity ringdown technique is a measurement of the decay of light intensity within a high-finesse optical cavity. In a typical measurement, a pulse of light is directed along the cavity axis towards the back face of one of the mirrors, and the small amount of light coupled through the mirror into the cavity undergoes repeated reflections between the two mirrors. A small fraction of light is lost on each round-trip of the cavity, primarily due to transmission or absorption by the mirrors or absorption or scattering within the cavity, with the result that the light intensity within the cavity decays exponentially with time. The exponential decay may be recorded by positioning a sensitive photodetector behind the second mirror to detect the light leaking out through the mirror on each pass. The time constant, $\tau$, of the decay, also known as the 'ringdown time' is given by

$$
\tau=\frac{n d}{c(L+\alpha C l)}
$$


where $d$ is the cavity length, $c / n$ the speed of light within the cavity ( $n$ is the refractive index of the cavity medium), $L$ the cavity loss per pass in the absence of a sample, and $\alpha$ the absorption coefficient of an absorbing species present at concentration $C$ over a path length $l$ within the cavity. Because the measured decay constant is independent of the absolute intensity of light injected into the cavity, CRDS is largely insensitive to noise caused by shot-to-shot fluctuations in the intensity of the pulsed laser source.

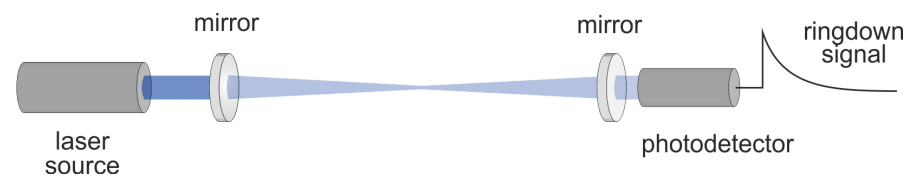

Fig. 1 Schematic of the experimental setup for a cavity ringdown or cavity-enhanced absorption spectroscopy measurement.

For a gas-phase measurement, the cavity loss $L$ for a simple two-mirror cavity is simply given by $1-R$, with $R$ the mirror reflectivity. For liquid-phase experiments, $L$ also includes refraction, reflection and scattering losses from the (ideally nonabsorbing) solvent and any container surfaces introduced into the cavity. Equation (2) is valid both for two-mirror cavities and for the fibre loop cavities considered in Section 3.1 and Chapter [CROSS REFERENCE CHUJI WANG CHAPTER]. Note that apart from constants of the experimental setup, such as the length of the cavity, the intrinsic cavity losses, and the path length through the sample, the only parameters that determine the ringdown time are the absorption coefficient and concentration of the sample. Consequently, by recording the ringdown time in the presence and absence of the sample (denoted $\tau$ and $\tau_{0}$, respectively), the absorption per unit path length, $\kappa=\alpha C$, can be determined, yielding the concentration $C$ if the absorption coefficient is known at the wavelength of interest.

$$
\kappa=\alpha C=\frac{n d}{c l}\left(\frac{1}{\tau}-\frac{1}{\tau_{0}}\right)
$$

\subsection{General principles of cavity-enhanced absorption spectroscopy}

Cavity ringdown measurements require the use of a pulsed laser or rapidly switched continuous wave $(\mathrm{CW})$ laser in combination with a fast detector and associated electronics in order to record the decay transient of the cavity. Cavity-enhanced absorption spectroscopy [37, 38, 39] (CEAS), also known as integrated cavity output spectroscopy (ICOS), is an experimentally simpler variant of cavity ringdown spectroscopy in which a CW (or pseudo-CW) light beam is coupled into the cavity, and the intensity coupled out from the cavity is monitored. It can be shown that the resulting signal is proportional to the ringdown time of the cavity $[38,40]$, and there- 
fore provides the necessary information on absorption without the need to record the decay transient. In many cases this experimental simplification is enough to make CEAS the preferred approach over CRDS. In addition, CEAS often has a larger dynamic range than CRDS. While in CRDS measurement, intense absorptions often result in a ringdown time that is too short to measure with sufficient accuracy [32], such absorptions do not pose a problem for CEAS measurements, in which only an intensity must be measured. As we shall see in Section 3.2, CEAS has a further advantage over CRDS when broadband measurements are desired. However, while CEAS has a number of advantages over CRDS, it also has two disadvantages. Firstly, CRDS yields an absolute absorption measurement, while CEAS provides only a relative absorption measurement unless a calibration measurement is carried out in order to determine the mirror reflectivity (often this is achieved in a separate ringdown measurement). Secondly, since the method involves a measurement of light intensity rather than rate of decay of intensity, the achievable signal-to-noise ratio is now dependent on the stability of the light source.

Assuming a low loss per pass, a valid approximation in any cavity-enhanced spectroscopy measurement, Fiedler et al. [41] showed that the absorption per unit path length for a CEAS measurement is given by

$$
\kappa=\frac{1}{d}\left(\frac{I_{0}}{I}-1\right)(1-R)
$$

where $d$ is the cavity length and $R$ the mirror reflectivity, as before, and $I_{0}$ and $I$ are the transmitted light intensity in the absence and presence of an absorbing sample.

Equations 4 and 8 indicate that a CEAS measurement is more sensitive than a single-pass absorption measurement by a factor of $(1-R)^{-1}$. This factor is equal to the number of passes through the sample during the measurement, and is usually referred to as the cavity enhancement factor [34](CEF). The CEF may be determined experimentally in any one of a number of ways; for example, by measuring the mirror reflectivity, as noted above, by making an absorption measurement on a sample with a known $\kappa$, or by measuring the ratio of the cavity-enhanced to single-pass absorption under equivalent experimental conditions.

\subsection{Detection limits in cavity ringdown and cavity-enhanced absorption measurements}

The detection limit in a cavity ringdown measurement is determined by the minimum measureable change in ringdown time, $\Delta \tau_{\min }$, on introduction of a sample to the cavity. Based on IUPAC recommendations [42], this is often defined quantitatively as three times the standard deviation in the baseline ringdown time, $\tau_{0}$. Once $\Delta \tau_{\min }$ has been determined, by making the (very good) approximation that at low sample concentrations $\tau \tau_{0} \approx \tau_{0}^{2}$, we can rearrange Equation (3) to determine the minimum detectable absorption per unit path length, $\kappa_{\min }$ [43]. 


$$
\kappa_{\min }=\frac{n d \Delta \tau_{\min }}{c l \tau_{0}^{2}}
$$

Inspection of Equation (5) reveals that to improve the sensitivity of a ringdown measurement, we can either minimise the baseline cavity losses in order to maximise $\tau_{0}$, or increase the path length travelled through the sample on each pass. When dealing with very small sample volumes, the scope for increasing the path length is limited, and improving the detection sensitivity relies primarily on minimising the cavity losses. Since the cavity length, and therefore the ringdown time, varies widely between different experiments, a more useful parameter for comparing the cavity losses associated with different experimental setups is the number of passes $N$ through the cavity per unit ringdown time.

$$
N=\frac{\tau_{0} c}{n d}
$$

Since signal is usually acquired over more than one ringdown time, the cavity enhancement factor (CEF) relative to a single-pass measurement is generally larger than $N$.

A similar approach can be used to determine the detection limit in a cavityenhanced absorption measurement. Making the (again, very good) approximation that

$$
\frac{I_{0}-I}{I} \approx \frac{I_{0}-I}{I_{0}},
$$

the minimum detectable absorption per unit path length is given by

$$
\kappa_{\min }=\frac{1}{d} \frac{\Delta I_{\min }}{I_{0}}(1-R)
$$

where $\Delta I_{\min }$ is the minimum detectable change in light intensity transmitted through the cavity, again taken to be three times the standard deviation in the baseline noise of the measurement.

\section{Applying cavity-enhanced spectroscopies to the liquid phase}

Achieving high detection sensitivity in cavity-enhanced spectroscopic measurements relies on keeping cavity losses attributable to anything but absorption by the species of interest to a minimum. Measurements on condensed-phase samples raise considerable challenges in this context. The most straightforward way to make measurements on liquid samples is to place the solution inside the cavity in direct contact with the mirrors. However, this requires a fairly large volume of liquid sample, which may not always be available, and also introduces high scattering losses. The much higher refractive index of liquids relative to air can alter the reflectivity properties of dielectric cavity mirrors, and in extreme cases, corrosive samples can cause permanent damage to the mirror surfaces. To avoid placing liquid in contact with 
mirrors, the sample is generally enclosed within a cuvette or other container. This introduces four additional surfaces into the cavity, and minimising scattering, refraction, and reflection losses at these surfaces is critical to maintaining the low overall cavity losses and correspondingly long ringdown times needed for high-sensitivity absorption measurements. A number of innovative cavity configurations have been employed for liquid-phase absorption measurements, and we will explore a variety of these in Section 3.1. A point worth noting is that because the round-trip cavity losses tend to be considerably higher in liquid-phase CRDS and CEAS measurements than in their gas-phase counterparts, the reflectivity of the mirrors is usually no longer the dominant factor determining the overall round-trip losses. As a result, lower-cost mirrors with somewhat lower reflectivity may often be used without significantly affecting the performance of the cavity.

A second consideration when making absorption measurements on liquids is the structure of the spectral absorption features for the molecule of interest. This is particularly true for sensing applications. While gas-phase species generally exhibit sharp absorption lines and detailed rovibrational structure in their absorption spectra, at least when probed at sufficiently high spectral resolution, intermolecular interactions in the liquid phase blur this structure into broad, relatively featureless absorption bands. For sensing applications in the gas phase, it is often sufficient to identify a single rovibrational absorption line that is distinct to the molecule of interest, and to record the absorption signal at the corresponding single wavelength. This is less likely to be possible in liquid-phase sensing applications, as it tends to be much more difficult to identify a single wavelength within an absorption band of the analyte of interest that does not overlap with absorption signals from solvent or other chemical species present in the sample. An alternative is to record the absorption spectrum over a range of wavelengths and employ a fitting procedure based on the known absorption features of the various chemical species present in the sample to extract the contribution from the species of interest. As in all absorption measurements, solvents must be chosen carefully, as common solvents such as water, methanol and ethanol often have significant absorption features in spectroscopic regions of interest. Historically, most CRDS and CEAS spectrometers have employed a tuneable monochromatic laser source to record absorption spectra over a range of wavelengths. The ringdown time or transmitted intensity is measured one wavelength at a time as the laser is scanned, and data acquisition rates are correspondingly rather slow. In more recent years, a considerable amount of effort has been invested into developing 'broadband' CRDS and CEAS methods. These employ a broadband light source and spectrally resolving detector, allowing the complete absorption spectrum over the wavelength region of interest to be recorded in a single measurement. Broadband cavity-enhanced spectroscopy methods are described in Section 3.2. 


\subsection{Cavity configurations for liquid-phase analysis}

A range of different cavity configuration that have been employed successfully for liquid-phase CRDS and CEAS measurements are illustrated in Figure 2. Configuration (a), in which the entire cavity is filled with the liquid sample, has already been discussed in Section 3. The high scattering losses occasioned by the liquid usually limit the cavity length in this configuration to a few millimetres, rather than the more usual dimensions of tens of centimetres. This reduces the ringdown time to such a degree that sub-nanosecond time resolution is required in order to make an accurate measurement in a CRDS measurement, though CEAS measurements are still feasible, and there have been some notable proof-of-concept studies employing this approach. Indeed, McGarvey et al [33] achieved the highest cavity enhancement factor of any liquid phase CEAS measurement reported to date in their measurements on Bacteriochlorophyll $a$ at $783 \mathrm{~nm}$, employing a cavity of length $1.7 \mathrm{~mm}$ formed from $99.998 \%$ mirrors. The authors even speculated that single-molecule detection may be possible within a very short cavity of length $\sim 10 \mathrm{mum}$. A more modest CEF of 429 was obtained by Seetohul et al. [36], who employed a $20 \mathrm{~cm}$ cavity formed from $99.9 \%$ mirrors in combination with an LED light source. This was still sufficient to detect methylene blue at the picomolar level. The CEF was lower than the value of around 1000 that was expected based on the known mirror reflectivity and cavity length, which the authors took to indicate a reduction in the mirror reflectivity on contact with the liquid sample.

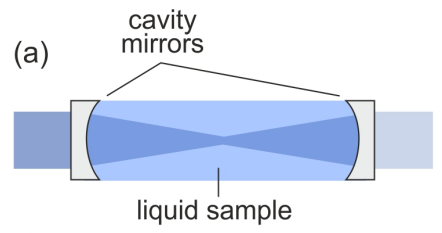

(b)

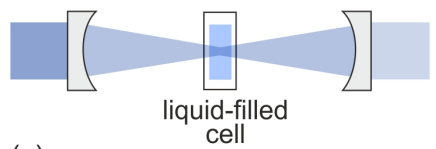

(c)

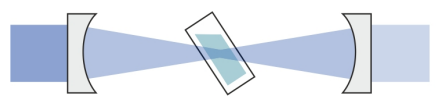

(e)

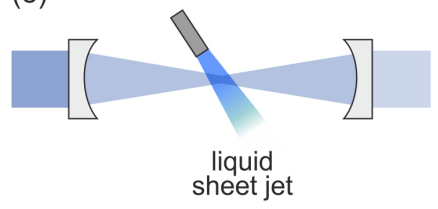

(f)

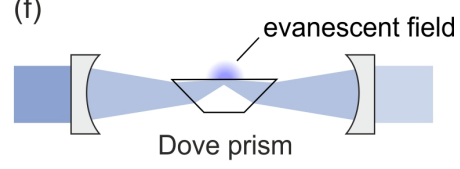

Fig. 2 Possible configurations for liquid phase CRDS and CEAS: (a) liquid-filled cavity; (b) normal-incidence absorption cell; (c) Brewster-angle absorption cell; (d) liquid sheet jet; (e) evanescent wave absorption. Adapted from reference [44]

Either one of two strategies may be adopted when introducing an absorption cell into a two-mirror cavity to contain a liquid sample. In the first (illustrated in Figure 2(b)), the cell is mounted at normal incidence to the laser beam, such that all 
reflections are retained along the cavity axis and reflection losses are therefore minimised. In the second (illustrated in Figure 2(c)), the cell is mounted at Brewster's angle, in which case the reflection losses at the surfaces are theoretically zero for light polarised parallel to the plane of incidence [27]. While either approach sounds appealling in theory, in practice there are problems with both. In the first geometry, all surfaces within the absorption cell must be parallel to each other to a high degree of precision in order to ensure that the light circulating within the cavity strikes all surfaces at exactly normal incidence. In practice, this is rarely the case, and some light will almost always be reflected into unstable cavity modes and be lost from the cavity on each encounter with the absorption cell. This problem can be mitigated by reducing the cavity length, which relaxes the alignment requirements to some extent, though at the cost of a reduced ringdown time. As an example, van der Sneppen et al. [29] investigated CRDS as a detection technique for liquid chromatography, inserting a cuvette into a short $(4 \mathrm{~cm})$ cavity at normal incidence to the beam axis, and demonstrated detection limits (in terms of absorption per unit path length) of around $10^{-4} \mathrm{~cm}^{-1}$ for a range of nitro-polyaromatic hydrocarbons. In the second geometry, the different refractive indices present at the four interfaces within the absorption cell mean that, while there is an 'optimum angle' at which surface reflection losses are minimised, there is in fact no single Brewster angle condition that is satisfied at all four interfaces. In addition, the optimum angle for the absorption cell depends strongly on the refractive index of the liquid sample, and the cell positioning within the cavity must therefore be reoptimised for each sample.

Reported CEAS measurements on liquids have relied almost exclusively on either the liquid-filled cavity or normal-incidence absorption cell configurations. However, for CRDS measurements, the Brewster-angle geometry has proved considerably more popular than the normal-incidence geometry. Xu et al. [28] have published one of the lowest detection limits, employing both the cavity geometry shown in Figure 2(c) and a second geometry in which two Brewster cells were incorporated into the cavity, and recording a minimum detectable absorption per unit path length of $10^{-7} \mathrm{~cm}^{-1}$ for the fifth vibrational overtones of the C-H stretch in pure benzene and benzene in solution with hexane. Snyder et al. [27] went to considerable lengths to optimise the Brewster-cell arrangement, designing a custom liquid flow cell which ensured that the Brewster angle condition was satisfied at every interface. Using a pulsed laser to excite the cavity, the resulting cavity enhancement factor was similar to that achieved by $\mathrm{Xu}$ et al, although the detection limit was significantly poorer due to a shorter path length through the sample on each pass, at $6.2 \times 10^{-4} \mathrm{~cm}^{-1}$. By replacing the pulsed laser with a narrowband $\mathrm{CW}$ diode laser [30], which was carefully mode matched into the cavity to excite a single cavity mode, the CEF was improved by more than a factor of two. This, together with the greatly improved signal-to-noise ratio achieved with the more stable light source, reduced the detection limit to $7.8 \times 10^{-6} \mathrm{~cm}^{-1}$.

An innovative approach which solves the problem of having liquid sample in contact with the mirrors, while at the same time eliminating the need to introduce a liquid container into the cavity, is the flowing liquid-sheet jet configuration developed by Alexander [31] and illustrated in Figure 2(d). The resulting cavity enhance- 
ment factor is similar to that of Xu et al. and the pulsed-laser work of Sneppen et al., but the very short path length per pass through the liquid jet of $23 \mu \mathrm{m}$ yields a relatively poorer detection sensitivity of $1.6 \times 10^{-2} \mathrm{~cm}^{-1}$. Also, though the probed volume using this approach is very small, the flowing jet requires a relatively large reservoir volume of sample.

All of the cavity configurations we have considered so far involve what might be termed 'direct' absorption measurements, in which the absorption is measured via direct transmission of the laser beam through the liquid sample. An alternative approach exploits the evanescent wave generated at a surface within the cavity where the light undergoes total internal reflection. A liquid or solid sample placed on the surface absorbs the evanescent field, leading to a reduction in the ringdown time of the cavity. An example of such an approach, in which the evanescent field is created at the surface of a Dove prism placed within the cavity, is shown in Figure 2(e), though numerous other configurations are possible [45, 46, 47], including some in which a custom-designed prism acts as both the optical cavity and the sample interface $[48,49,50]$. Since only a relatively small fraction of the propagating light interacts with the sample in such an arrangement, one might expect a considerable reduction in sensitivity relative to direct absorption methods. However, since cavity configurations for evanescent absorption measurements usually introduce fewer additional surfaces into the cavity than a liquid container, this is often compensated for by a reduction in the overall cavity losses relative to a direct absorption measurement. Because the evanescent wave only propagates a very short distance from the surface, on the order of a wavelength, cavity configurations of this type are well suited to the study of thin films and processes at interfaces [46, 47, 49, 50].

An increasingly popular type of cavity exploits total internal reflection within an optical fibre. Since optical-fibre cavities are the subject of Chapter [CHUJI WANG'S CHAPTER] of this book, we will only provide a brief description of such cavities here. Fibre cavities can be grouped into two classes, namely linear fibre cavities and fibre-loop cavities, both of which are illustrated schematically in Figure 3. Linear fibre cavities consist of a length of fibre with either a mirror-coating $[51,52,53]$ or a fibre Bragg grating [54] at either end to confine the light within the fibre. Light is injected into one end of the fibre and undergoes repeated reflections along the length of the fibre, with the waveguiding properties of the fibre ensuring that the cavity is permanently and robustly aligned. Linear fibre cavities almost exclusively employ evanescent field absorption: a sensing region is created by stripping the plastic coating from a small region of fibre and tapering the fibre either by etching in hydrofluoric or flurosilicic acid, or by heating and pulling, to expose the evanescent field of the waveguided light. Fibre-loop cavities [55, 25, 56] consist of a loop of optical fibre in which the excitation light circulates, with the sample probed by direct absorption, having been introduced into a small gap between the fibre ends where they join to form the loop. There are considerable challenges associated with optimising the side-coupling of light into and out of the cavity and minimising losses associated with the coupling and sample regions when using this type of cavity. There have been numerous demonstrations of fibre cavities for sensing of a range of properties, including temperature [57], pressure [58], stress [59] 
and refractive index [60]. In applications to chemical sensing, optical fibre cavities are well suited to the interrogation of extremely small sample volumes, being inherently size-matched to microfluidic channel widths, for example. They are also inherently broadband in nature, facilitating measurements at multiple wavelengths or across a continuum of wavelengths. However, fibre-based cavities are much more lossy than their mirror-based counterparts, such that their robust and compact nature is usually offset by significantly poorer detection limits.

(a)

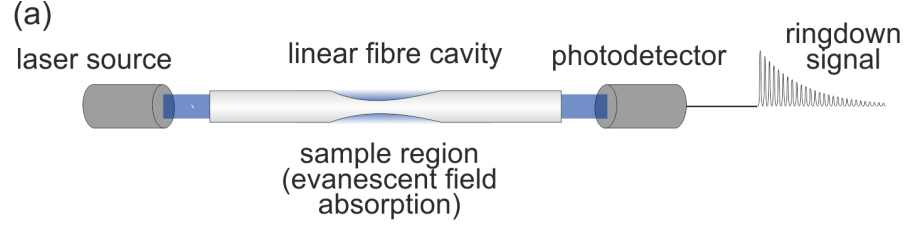

(b)

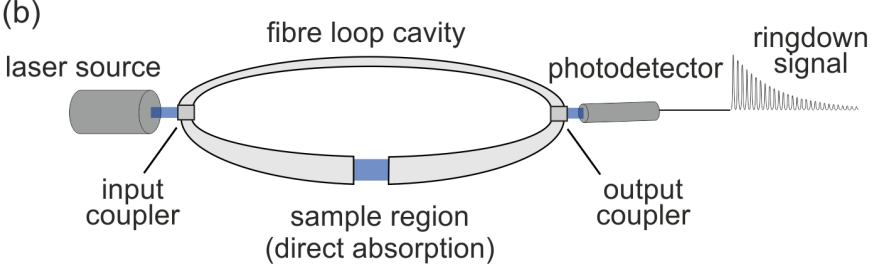

Fig. 3 (a) Linear fibre cavity, employing either mirror-coated fibre ends or fibre Bragg gratings to confine the light within the cavity; (b) Fibre-loop cavity, with input and output coupling from the side of the optical fibre.

\subsection{Broadband cavity ringdown techniques}

As noted earlier, the ability to perform absorption measurements across a range of wavelengths is extremely useful in the context of the broad absorption features associated with a liquid phase analyte. Under these conditions, measurements at a single wavelength provide limited information, and absorption by other species present in the sample is often problematic. By recording across a complete absorption band, broadband measurements can provide specificity, and in the case where several absorbing species are present, they can provide the necessary spectral coverage to monitor multiple species simultaneously.

There have been a number of approaches to combining a broadband light source with cavity ringdown measurements [21]. Most early methods lacked a broadband detector and used a monochromator to scan the wavelength either at the entrance to or exit from the cavity, making them essentially equivalent to experiments carried out with a tuneable (rather than broadband) light source. A variation on this approach was the Fourier transform CRDS method developed by Engeln and Meijer 
[61], which built up the wavelength-resolved ringdown signals from a series of interferograms. Engeln and coworkers later developed a phase-shift version of the technique [62] with significantly higher sensitivity. All of these approaches essentially involve a wavelength scan. An alternative approach is to record all wavelengths simultaneously and perform a scan in time to build up the ringdown transients at each wavelength. This was the approach adopted by Czyzewski [63], who dispersed the broadband light through a spectrograph at the cavity exit and imaged the resulting spectrum using a time-gated CCD camera. By sweeping the CCD time gate through the ringdown decay over consecutive laser pulses, the broadband ringdown trace is obtained. This method neatly makes use of the broadband character of the light source, but because the ringdown trace is built up over multiple laser shots, any shot-to-shot fluctuations in the intensity of the light source manifest as additional noise on the ringdown traces.

There are only very few examples of 'true' broadband cavity ringdown spectroscopy, in which the ringdown decay transient is recorded for each wavelength on every laser shot. The first of these is a technique known as 'ringdown spectral photography', developed by Scherer $[64,65]$ and illustrated schematically in Figure 4(a). Broadband radiation leaving the cavity is incident first on a rotating mirror, and then on a diffraction grating, before being imaged on a two-dimensional CCD array. The rotating mirror resolves the time-dependence of the signal, with light from different time segments of the ringdown decay striking different points on the diffraction grating. The diffraction grating then disperses the signal in wavelength along a perpendicular axis. The resulting two-dimensional image is resolved in time along one axis and wavelength along the second axis. The resolution of the grating and CCD array used in these measurements yielded a spectral resolution of around $1.5 \mathrm{~cm}^{-1}$, sufficient to resolve rotational structure, while the time resolution, determined by the rotation speed of the mirror and the number of pixels in the CCD sensor, was typically around $40 \mathrm{~ns}$ per pixel. The second broadband CRDS technique, developed by Ball et al. [66, 21], also employs a grating to disperse the broadband light in wavelength as it emerges from the cavity. However, the time resolution is achieved using a two-dimensional clocked $512 \times 1024$ CCD array (see Figure 4(b). The CCD array is masked so that the dispersed cavity output falls only on the first ten rows of pixels. After acquiring signal for $5 \mu \mathrm{s}$, the pixel voltages are clocked such that the charge collected on these rows is transferred to the next ten rows, allowing a new image to be recorded on the (now empty) first ten rows. The process is repeated, shunting the acquired charge further down the sensor every $5 \mu \mathrm{s}$. This procedure permits around ten images to be recorded during the $\sim 50 \mu$ s ringdown decay, with the final image read out from the CCD sensor comprising a two-dimensional 'wavelength vs time' image similar to that recorded in the ringdown spectral photography method. Following initial proof of concept work [66, 21], the instrument was used in field measurements during the 2002 North Atlantic Marine Boundary Layer Experiment [67] to measure ambient levels of $\mathrm{NO}_{3}, \mathrm{~N}_{2} \mathrm{O}_{4}, \mathrm{I}_{2}$ and OIO.

The need to achieve both wavelength-resolved and time-resolved detection makes broadband cavity ringdown measurements experimentally complex. In contrast, broadband CEAS requires only a broadband light source and a means of wave- 

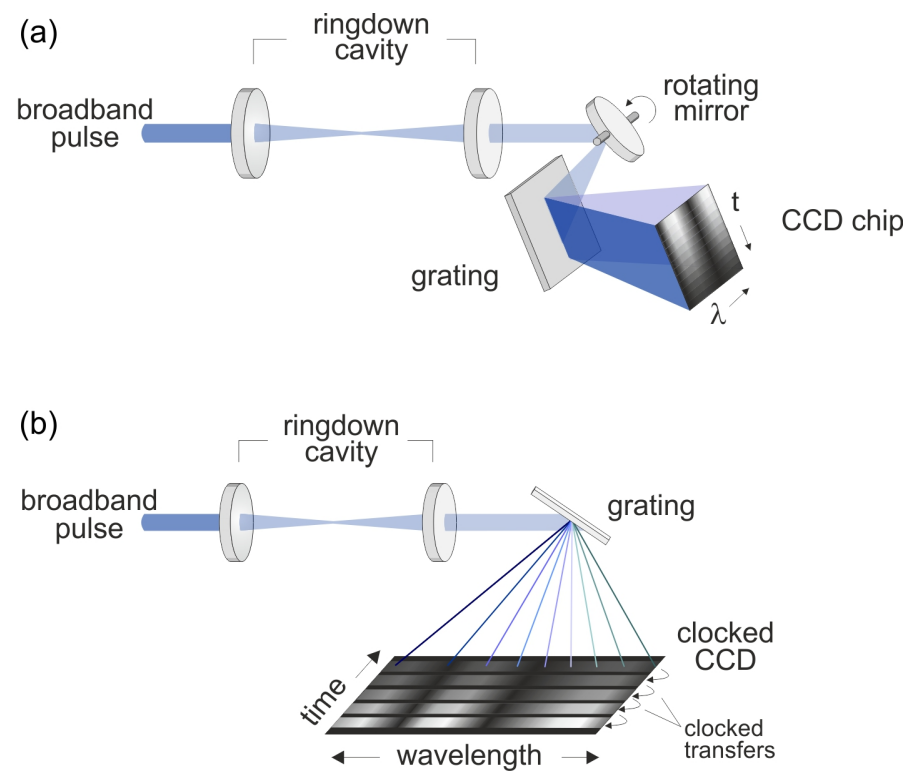

Fig. 4 (a) Ringdown spectral photography; and (b) clocked CCD camera approaches to broadband cavity ringdown spectroscopy. Adapted from reference [68].

length dispersion of the cavity output, often a commercial spectrograph or spectrometer. It is therefore experimentally much more straightforward than the corresponding CRDS technique, and has been adopted enthusiastically by the cavityenhanced spectroscopy community for high-sensitivity broadband absorption measurements. A variety of light sources have been used for broadband CEAS measurements, including Xe arc lamps [41, 69, 70, 71, 72, 73, 74], light emitting diodes $[75,76,77,78,79,80,81]$, supercontinuum sources [82, 83, 84, 85, 86, 87, 88], and femtosecond frequency combs $[89,90,91,92]$. Extremely high detection sensitivities have been achieved, down to the pptv level in some cases [71, 74, 77, 80, 81, 82], with signal averaging times of seconds to minutes depending on the intensity of the light source.

\section{Example application: microfluidic sensors}

There is currently a great deal of research aimed at developing microfluidic devices to carry out chemical processes. Known by a variety of names, including 'lab on a chip' and ' $\mu$ TAS' (micro-total-analysis systems), such devices integrate a raft of laboratory functions, such as sample preparation, mixing, reaction, and separation, onto a glass or plastic chip a few centimetres in size. There are many situations in which miniaturising chemical analysis in this way holds a number of advantages 
over bulk processing of chemical samples. A microfluidic platform is ideal when attempting to develop portable chemical tests, for example, and such chips have considerable potential for applications such as medical diagnostics, forensics, and environmental monitoring. Fabrication costs are generally low, and only very small volumes of sample and reagents are required for analysis, which is often particularly important when dealing with biological samples. A secondary advantage of the small reagent volumes employed is improved safety relative to lab-based tests. There is also the potential for very high-throughput chemical processing, achievable by running many experiments, reactions, or analyses in parallel on an array of chips. However, there are considerable challenges associated with developing such devices, not least the difficulty associated with the quantitative detection of chemical species in volumes on the order of picolitres. Cavity-enhanced spectroscopic techniques provide one potential approach to meeting this challenge [44].

As noted in Section 1, once path lengths are reduced to microfluidic dimensions of typically $50-100 \mu \mathrm{m}$, the absorption signal often becomes too small to measure reliably using conventional single-pass absorption spectroscopy. Many researchers have attempted to address this issue with a range of extended-path length singlepass methods [93, 94, 95, 96, 97, 98, 99, 100, 101, 102, 103, 104], and a variety of multi-pass microfluidic absorption cell designs [105, 106, 107, 108, 103, 109, $110,111,112,113$ ], many have which have recently been reviewed [44]. However, both of these approaches come at the expense of a larger sample volume, and to implement them in a particular application requires fairly major modifications to the microfluidic chip design. In contrast, embedding a microfluidic chip within an optical cavity (or, alternatively, embedding an optical cavity within a microfluidic chip) provides a large increase in path length, and a corresponding enhancement in detection sensitivity, with no accompanying increase in the probed volume of sample.

Incorporating an optical cavity into a microfluidic chip is equivalent to cavity configuration (a) of Figure 2, and avoids many of the problems outlined in Section 3.1 associated with incorporating a liquid container into the cavity. In this approach, the very short cavity length precludes ringdown measurements, but CEAS measurements are still possible. The simplest approach to creating a cavity within a chip is simply to mirror-coat the internal surfaces of a microchannel. This was the method adopted by Billot et al. [114], who coated $150 \mathrm{~nm}$ thick gold mirrors onto the inner surfaces of two glass substrates and then bonded them with PDMS (polydimethylsiloxane) to form a microfluidic chip containing a $50 \mu \mathrm{m}$ channel. Using the focused output from a halogen lamp as the light source and coupling the cavity output to a spectrometer, the spectrum of Rhodamine B was acquired over the wavelength range from $500-600 \mathrm{~nm}$, with a measured cavity enhancement of 50 relative to an uncoated channel. Further development in integrated-cavity microfluidic chip designs may well be inspired by advances in microfluidic laser technology $[115,116,117]$, with both applications sharing the requirement for a high-finesse on-chip optical cavity. Cavity designs for microfluidic lasers include ring dye lasers [118], and mirror-coated optical fibres positioned either side of one or more microfluidic channels [119]. By incorporating two microfluidic channels within the 
cavity, it has already been demonstrated by Galas et al. [119] that the latter design may be used for chemical sensing. The first channel was filled with Rhodamine 6G to provide laser gain, and the second with the analyte of interest. The sensitivity of the laser output to the loss introduced to the cavity by the absorbing analyte allowed the detection of micromolar solutions of methylene blue within an optical path length of only $140 \mu \mathrm{m}$.

An alternative to incorporating a cavity into a chip is to insert the microfluidic chip into a conventional two-mirror cavity. While the presence of a chip within the cavity increases the cavity losses, this approach has the advantage that a single cavity can be used to make measurements on a variety of different microfluidic chips and provides a fairly general platform for absorption measurements on microfluidic samples [120]. Any microfluidic chip to be used in such measurements must introduce the lowest possible optical loss to the cavity, and must therefore have a high degree of optical transparency and low surface roughness, with all surfaces within the chip fabricated to be as parallel as possible. It is also important that the beam waist within the cavity is carefully matched to the size of the microfluidic channel to be probed, in order to minimise scattering losses. Using a chip fabricated from glass slides sandwiched together with thiolene optical adhesive, inserted at Brewster's angle within a two-mirror optical cavity, James et al carried out a series of proof-of-concept experiments to demonstrate the capabilities of CRDS in monitoring both static and time-varying analyte concentrations, with a cavity enhancement factor of around 150. The minimum detectable absorption per unit path length was determined from a series of measurements on $\mathrm{KMnO}_{4}$ solutions of varying concentrations to be $1.0 \times 10^{-3} \mathrm{~cm}^{-1}$. Measurement of $\mathrm{pH}$ to a precision of $\pm 0.04 \mathrm{pH}$ points was demonstrated on a $20 \mathrm{~nL}$ sample by monitoring the absorption of phenolphthalein indicator present at millimolar concentrations in a series of buffered $\mathrm{pH}$ standards. A typical titration curve from these measurements is shown in Figure 5(a). Finally, the Belousov-Zhabotinsky oscillating reaction was followed on-chip to demonstrate the potential of CRDS for chemical kinetics studies. The reaction involves the catalytic oxidation and bromination of malonic acid, and in the presence of a ferroin indicator, an oscillating red to blue colour change is observed with a period of several tens of seconds. The time-dependent absorption of the reaction mixture at $532 \mathrm{~nm}$ allows the oscillation to be followed on-chip in real time. Figure 5(b) shows a data set comparing the microfluidic measurements with a set of measurements made on a bulk reaction mixture using a conventional UV/vis spectrometer. While the oscillation period is similar in both cases, the shapes of the time-resolved absorption peaks are quite different, implying that the detailed reaction kinetics depend on the size and shape of the reaction vessel. At later times the microfluidic measurements were degraded somewhat by the formation of bubbles of $\mathrm{CO}_{2}$ (one of the reaction products) within the microfluidic channel. In principle, CRDS offers the possibility of tracking chemical kinetics down to the microsecond timescale of the ringdown decay and, if a time-varying concentration is allowed when fitting the ringdown traces to determine the absorption, to even shorter timescales.

The discussion of microfluidic samples so far has focused on continuously flowing liquids. However, many microfluidic applications rely on the generation and ma- 

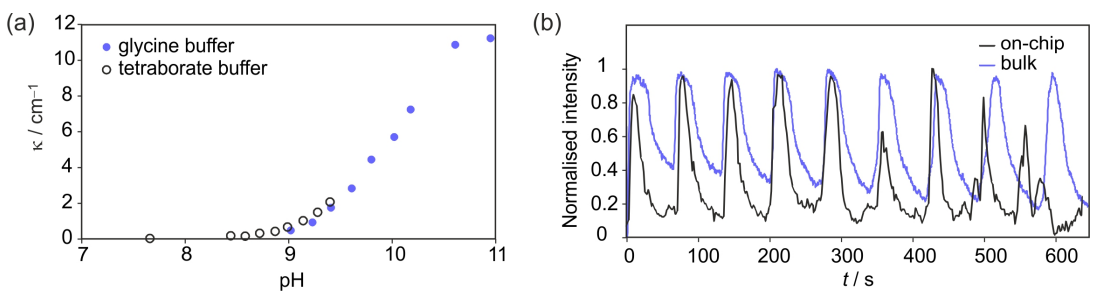

Fig. 5 (a) Optical measurement of $\mathrm{pH}$ on a microfluidic chip via CRDS. The measured absorption at $532 \mathrm{~nm}$ of an indicator dye for a series of buffered $\mathrm{pH}$ standards tracks the titration curve of the indicator; (b) Reaction monitoring on a microfluidic chip. Oscillatory behaviour of the BelousovZhabotinsky reaction monitored via single-pass absorption in a $1 \mathrm{~cm}$ cuvette (grey) and CRDS on a microfluidic chip (black). Adapted from [120].

nipulation of a sample consisting of uniform droplets, which essentially constitute miniature 'test tubes' with volumes of femtolitres to picolitres. Droplet microfluidics has been used for applications ranging from synthesis of nanoparticles [121] and optimisation of protein crystallisation conditions [122] through to reaction kinetics studies [123, 124, 125, 126]. Both CRDS and CEAS have sufficient sensitivity for single-droplet analysis [127, 128], and by making measurements as both the droplets and the carrier phase pass through the beam, the carrier phase measurement can be used as the 'blank' for the absorption measurement, assuming any effects of refractive index mismatch between the two phases can be accounted for. The results of proof-of-principle investigations on dye droplets using both CRDS at $532 \mathrm{~nm}$ [120] and broadband CEAS [85] are shown in Figure 6. While in both studies the data acquisition systems employed were relatively slow, limiting the studies to large droplets (or 'slugs') and low droplet production rates, with suitably fast data acquisition hardware it should be entirely feasible to study considerably smaller droplets at high droplet production rates. In both cases the signals are characterised by a large reduction in measured ringdown time or transmitted intensity as the boundaries of each droplet pass through the laser beam. This can be attributed to the lensing effect of the droplet meniscus scattering light out of the cavity. In the centre of the droplet, the absorption signal of the droplet contents is recovered. In the case of the broadband CEAS measurements, the entire absorption spectrum of the aqueous $\mathrm{K}_{2} \mathrm{IrCl}_{6}$ dye used for the measurements is measured. For the CRDS measurements, the chip was placed at Brewster's angle within the cavity, and the observed ringdown times depend both on absorption by the droplets and carrier solution, and also on the refractive indices of the two solutions, since these determine the optimum 'Brewster' angle of the chip within the cavity.

Finally, there has been considerable interest in using optical-fibre-based cavities for microfluidic measurements. The serendipitous size matching between the diameter of an optical fibre and a microfluidic channel makes the use of opticalfibre cavities in microfluidic sensing an attractive prospect. Fibre-based cavities fall into two categories: linear fibre cavities [51, 52, 53, 54]; and fibre-loop cavities $[25,56]$. These are shown schematically in Figure 3. Fibre cavities are covered in detail in Chapters [CROSS-REFERENCE] and [CROSS-REFERENCE] of this 
(a)

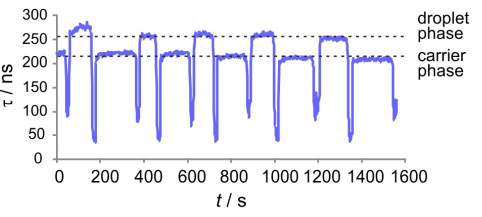

(b)

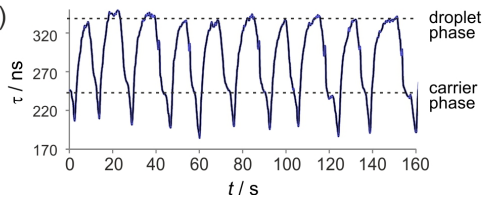

(c)

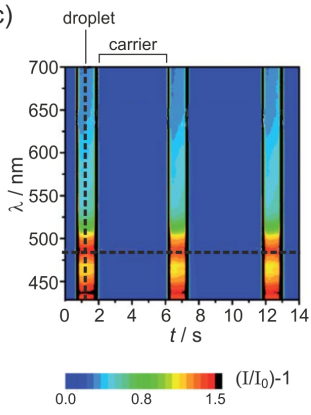

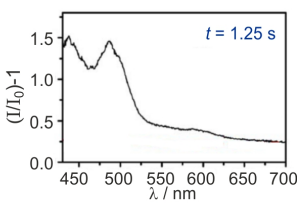

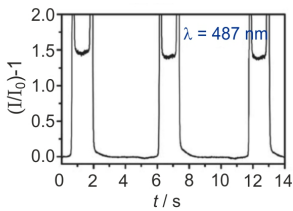

Fig. 6 Droplet flow analysis via CRDS of slugs of $1 \mu \mathrm{M}$ aqueous Rhodamine $6 \mathrm{G}$ in a toluene carrier phase at a flow rate of (a) $0.008 \mathrm{~mL} \mathrm{~min}^{-1}$, and (b) $0.075 \mathrm{~mL} \mathrm{~min}^{-1}$ on a glass-thiolene chip placed at Brewster's angle within the cavity; (c) Droplet flow analysis via broadband CEAS of slugs of $40 \mu \mathrm{M}$ aqueous $\mathrm{K}_{2} \mathrm{IrCl}_{6}$ dye solution in a toluene carrier phase. The cuts through the full data set show the absorption spectrum of a single droplet and the time dependence at the peak of the absorption curve of the dye. Adapted from [120] and [85].

book, so only a very brief overview will be given here, with the focus on applications in microfluidics.

As noted previously in Section 3.1, fibre cavities intrinsically probe extremely small sample volumes, and are inherently broadband in nature, allowing measurements to be made at multiple wavelengths or across a continuum of wavelengths simultaneously. Unfortunately, the compact and robust nature of such cavities tends to be offset by the fact that the optical losses are much higher than for a two-mirror cavity, typically of the order of a few percent, such that the minimum detectable absorption per unit path length is often significantly lower. While the fibre itself occasions small losses due to imperfect transmission, the majority of the optical losses within a fibre-loop cavity are associated with the sample region and the couplers used to couple light into and out of the loop. As a consequence, it is possible to achieve relatively long ringdown times even with relatively high losses on each pass simply by increasing the length of fibre used to create the loop cavity, noting that it takes the circulating light around $5 \mathrm{~ns}$ to traverse $1 \mathrm{~m}$ of fibre. The ability to achieve long ringdown times in this way makes fibre-loop cavities well suited to phase-shift[129], rather than direct, measurements of the ringdown time, and this approach has been implemented with some success by Loock and coworkers $[130,131,132,133]$. Phase-shift measurements employ a CW laser source modulated at a frequency $\omega$, and use a lock-in amplifier to record the phase shift introduced by the cavity to the modulated light coupled out from the cavity. The phase shift is related to the ringdown time $\tau$ and the modulation frequency $\omega$ by

$$
\phi=-\arctan (\omega \tau)
$$


By injecting light into the cavity of multiple wavelengths, each with a different modulation frequency, the phase-shift approach allows absorption measurements to be made at two or more wavelengths simultaneously [133].

Whether making a direct measurement of the ringdown time or employing phaseshift techniques, the overriding aim when employing fibre cavities in microfluidics (or any other application) is to minimise the losses associated both with the sample region and with the light coupling scheme used to couple light into and out of the fibre. In linear fibre cavities, light may be coupled through a mirrored fibre end in much the same way as for a two-mirror cavity, while in a fibre-loop cavity the excitation light must be coupled into the loop through the side of the fibre, a much more challenging prospect. A variety of side-coupling schemes have been developed, including bend coupling [55], use of commercially available 99:1 coupler/splitters [134], and methods exploiting microfabricated mirrors machined into the fibre core $[135,136]$.

The simplest way to incorporate a microfluidic sample into a fibre cavity is to introduce a break into the optical fibre and align the two fibre ends on either side of a microfluidic channel. In order to maintain a low optical loss, in the absence of an absorbing species it is important that virtually all of the light emitted from the first fibre end is coupled back into the second fibre end. In practice this is very difficult to achieve for gaps larger than a few tens of microns [55], and divergence of the light emitted from the first fibre end means that end separations are limited to distances on the order of the fibre core diameter. A modest improvement in the achievable path length per pass can be obtained by lensing the ends of one or both fibres, such that light emitted from the first fibre is focussed through the sample into the second fibre. Achieving the highest detection sensitivity relies on finding the best compromise between minimising the loop losses by optimising the coupling scheme and keeping the width of the sample gap as small as possible, and maximising the path length through the sample on each pass by increasing the gap width. Depending on the relative balance between these two factors, cavity enhancement factors ranging from 50 or so $[55,136]$ down to less than $5[137,138]$ have been obtained. The highest cavity enhancement factors allow the detection of a variety of analytes down to submicromolar concentrations in a detection volume of tens of nanolitres [133, 136].

An alternative approach to improving the sensitivity of fibre-loop cavity ringdown measurements is to accept that the loop losses will always be relatively large in comparison with two-mirror cavities, and to attempt to compensate for the losses by introducing a variable gain element into the loop. While this has not yet been implemented for microfluidic measurements, the approach has been investigated by Stewart et al [139] in fibre-loop ringdown measurements on gas-phase samples. Incorporating a $5 \mathrm{~cm}$ open path micro-optical gas cell into a $58 \mathrm{~m}$ fibre loop occasioned an insertion loss of around $20 \%$ per pass, which was offset by including an erbium-doped fibre amplifier (EDFA) into the loop. The optical gain element allows the ringdown time to be increased from around 100 ns to several tens of microseconds, but unfortunately the less than optimal stability of the amplifier (and therefore the ringdown time) becomes the limiting factor in performing sensitive absorption measurements [140]. Improving the amplifier stability is not trivial, but if the sta- 
bility issues can be addressed then amplified fibre-loop cavities offer considerable potential for making highly sensitive absorption measurements on microfluidic samples.

\section{Example application: sensing of trace compounds in water}

In the previous section, we outlined the general approaches employed for cavityenhanced spectroscopic measurements on microfluidic samples, and some of the important experimental considerations associated with such measurements. We now focus on a specific application, namely the quantitative determination of trace compounds in water. Such determinations are important in a range of contexts, including water quality testing and environmental monitoring, and there is considerable interest in the development of compact, robust sensors capable of operating remotely for long periods of time without human intervention.

One of the key applications for such sensors is in chemical oceanography. Understanding the rich chemistry of the world's oceans requires measurements of the distribution and dynamics of a broad range of elemental and molecular chemical species. In addition to probing the fundamental physical, thermodynamic, and kinetic properties of chemical species in marine environments, understanding the interactions between ocean chemistry and other biological, geological, and physical processes is of vital importance in understanding the past evolution of the earth, thereby allowing us to model its future. Several quantities of interest in chemical oceanography, such as temperature and salinity, are relatively straightforward to measure, and the technology for measuring them is highly advanced. As an example, a network of over 3000 research buoys making up the Argo float project continuously monitors temperature, pressure and salinity within the upper $2000 \mathrm{~m}$ of the ocean, transmitting data back to shore by satellite [141]. For other quantities, the development of suitable sensing systems is still very much a work in progress. As an example, photosynthetic phytoplankton require a range of nutrients (e.g. $\mathrm{NO}_{3}^{-}$ , $\mathrm{PO}_{4}^{3-}$, and $\mathrm{SiO}_{2}$ ) and micronutrients (mostly trace metals) in order to synthesise their cellular machinery. Phytoplankton play an important role in the synthesis of the majority of marine organic compounds from aquatic carbon dioxide, and represent a considerable $\mathrm{CO}_{2}$ sink; so much so that seeding oceans with the micronutrient $\mathrm{Fe}^{3+}$ in order to encourage the formation of phytoplankton ('algal') blooms is under serious consideration as a route to $\mathrm{CO}_{2}$ sequestration from the atmosphere [142]. To further our understanding of such processes there is an urgent need for robust chemical sensors suitable for field studies

Sensors based on microfluidic platforms represent an attractive approach due to their small size and low power and reagent consumption. However, as noted previously, a sensitive detection technique is required in order to detect low concentrations of analytes in volumes of nanolitres or less. The problem is compounded by the fact that many of the analytes of interest do not have any characteristic absorption features in spectroscopic regions for which suitable light sources are available. 
In the following, we will explore one possible solution to this problem, in which cavity-enhanced spectroscopies are combined with colourimetry in order to achieve selective and highly sensitive detection of a range of analytes.

Colourimetric assays employ a chemical reaction to convert the (non-absorbing) analyte of interest selectively into an intensely coloured derivative, which can then be quantified via an absorption measurement. Knowledge of the stoichiometry of the colourimetric reaction then allows the concentration of the initial analyte to be determined. The large absorption coefficient of the derivative compound provides a large signal amplification, which can be amplified further by employing cavity ringdown or cavity-enhanced absorption spectroscopy to make the absorption measurement. Colourimetric assays have already been developed on a microfluidic platform for analytes such as haemoglobin [104], botulinum toxin [143], and glucose [144, 145], amongst others, using a single-pass absorption measurement in the detection step.

To demonstrate the potential applications of cavity-enhanced spectroscopic detection for water sensing, we review the results of recent proof-of-concept experiments aimed at determining the detection limits for nitrite and iron using colourimetric CRDS. Nitrite $\left(\mathrm{NO}_{2}^{-}\right)$plays an important part in the global carbon and nitrogen cycles, as well as being a key marine nutrient required for phytoplankton growth $[146,147]$. At high levels, it is toxic to humans and animals, and its levels are carefully monitored in waterways and drinking water. The conentration of nitrite in sea water ranges from 0.1 to $50 \mu \mathrm{M}$, and the World Health Organisation guidelines set limits for short-term exposure to nitrites at $62.5 \mu \mathrm{M}$ [148]. The second analyte investigated, iron, is essential for all known living organisms [149]. The concentration of dissolved iron $\left(\mathrm{Fe}^{2+}\right.$ and $\mathrm{Fe}^{3+}$ ) varies widely in marine and aquatic environments, depending primarily on the dissolved oxygen concentration. In rivers, the concentration ranges from around 3 to $25 \mu \mathrm{M}$ [148], while in oceans the concentration is much lower, at around $0.02-2 \mathrm{nM}$ [149].

Two different colourimetric assays were used for detection of nitrite and $\mathrm{Fe}(\mathrm{II})$, respectively. The coloured products of both assays absorb strongly at $532 \mathrm{~nm}$, the wavelength of the frequency-doubled microchip Nd:YAG laser used for the CRDS measurements. Nitrite was detected via the Griess assay [150], which involves acidifying the nitrite sample, diazotising the nitrite with a primary amine (e.g. sulfanilamide), and coupling the product to an aromatic molecule to produce an intensely coloured azo dye with an absorption maximum $[151,152]$ in water of around $80,000 \mathrm{M}^{-1} \mathrm{~cm}^{-1}$ at $520 \mathrm{~nm}$. The dye is formed highly specifically in a 1:1 ratio from nitrite in the sample, so a quantitative absorption measurement on the dye yields the concentration of nitrite in the original sample. Iron concentrations can be determined colourimetrically using any one of a number of complexation reactions $[153,154,155]$, and for these measurements, complexation of $\mathrm{Fe}^{2+}$ with 4,7diphenyl-1,10-phenanthroline[156] (bathophenanthroline), which reacts with $\mathrm{Fe}(\mathrm{II})$ in a 3:1 ratio, was chosen due to the large absorption coefficient $\left(51,600 \mathrm{M}^{-1} \mathrm{~cm}^{-1}\right)$ of the coloured product at $532 \mathrm{~nm}$.

For the absorption measurements, the products of the colourimetric reaction are contained within a commercial short $(1 \mathrm{~mm})$ path length fused silica flow cell positioned at Brewster's angle within a conventional two-mirror cavity. Recall from 
Section 2.1 that determination of an absolute absorption per unit path length, $\kappa$, from a cavity ringdown measurement requires the measurement of two ringdown times, $\tau$ and $\tau_{0}$, corresponding to the sample of interest and a 'blank'. An important consideration in colourimetric CRDS measurements is the correct choice of blank for the $\tau_{0}$ measurement. An obvious choice for the blank would appear to be the deionised water used to make up the standard solutions of nitrite and iron. However, even the highest quality deionised water is likely to contain small but significant concentrations of the analyte of interest, and in fact a more appropriate blank is the reaction mixture obtained when the appropriate colourimetric reaction is carried out on a sample of deionised water. In practice, when dealing with extremely low concentrations of analyte, the quality of the blank solution is often one of the key limiting factors in determining the detection sensitivity, and the choice of blank should be given very careful consideration.

The minimum detectable absorbance per unit path length, $\kappa_{\min }\left(=\alpha C_{\min }\right)$, determined from equation 5 , with $\Delta \tau_{\min }$ taken to be three times the standard deviation in repeated measurements of the baseline $\tau_{0}$, was found to be around $1 \times 10^{-4} \mathrm{~cm}^{-1}$. Using the known absorption coefficients, $\alpha$ for the absorbing species, this yielded limits of detection of $1.4 \mathrm{nM}$ for nitrite and $3.8 \mathrm{nM}$ for Fe(II). A somewhat higher detection limit of $16 \mathrm{nM}$ for $\mathrm{Fe}$ (II) was obtained when the measurements were repeated in a $0.1 \mathrm{~mm}$ path length flow cell, a path length commensurate with a microfluidic channel. The detection limits are comparable with or better than those achieved previously in experiments employing much longer path lengths, and therefore requiring much larger sample volumes $[157,158,152,159,160,161,162,163]$. To put the results into context, when the illuminated volume and path length per pass within the flow cell is taken into account, we find that the measured limits correspond to the detection of around one billion molecules.

This case study demonstrates that colourimetric CRDS may be used to detect both nitrite and iron in path lengths similar to those encountered in microfluidic systems, and that with further development this technique could potentially form the basis for ship-based or remote sensors for monitoring trace species in both marine and aquatic environments. The sensitivity to nitrite is already sufficient for monitoring ambient levels in all such environments, and while further improvements are required in order to satisfy the requirements for monitoring Fe(II) in the open ocean, the sensitivity is already sufficient to monitor concentrations in river or drinking water. Incorporating a pre-concentration step into the analysis, while complicating the analytical procedure, would in principle allow ambient marine concentrations to be detected. Such steps have been demonstrated previously by a number of researchers [157, 164, 165], yielding sub-nanomolar limits of detection.

\section{The future}

We hope to have shown in this chapter that, while they do not achieve the often staggeringly high cavity enhancement factors seen in gas-phase measurements, cavity- 
enhanced absorption measurements on liquid samples provide a valuable means of improving the detection sensitivity, particularly in situations where the path length or sample volume are restricted. There is considerable scope for further improvement in sensitivity, and we conclude this chapter with a brief consideration of the various approaches that may be taken to achieve this.

Detection sensitivity in a cavity-enhanced spectroscopy measurement can be improved either by reducing the cavity losses, thereby increasing the ringdown time; by increasing the path length through the sample on each pass; or by reducing the shot-to-shot variation in the measured ringdown time. We will consider each of these factors in turn.

The cavity losses $L$ (in the absence of a sample) may be reduced either by increasing the mirror reflectivity or by reducing the losses associated with the microfluidic chip or other liquid container within the cavity. Inspection of Equations (5) and (2) reveals that the minimum detectable absorption per unit path length is proportional to $L^{2}$. As an example, the $1 \mathrm{~mm}$ flow cell used in the trace detection experiments described in Section 5 introduced a loss per pass of around $0.22 \%$, similar to the $0.2 \%$ loss introduced by the the $99.8 \%$ reflecting mirrors. If the mirrors were replaced with the 'best available' $99.999 \%$ reflectivity mirrors, and the flow cell was replaced by the custom-designed Brewster-angle flow cell developed by Snyder and Zare [27] (see Section 3.1), which has a reported loss of $0.06 \%$, then the cavity transmission would be increased from $99.58 \%$ to $99.94 \%$, the loss per pass would be reduced from $0.42 \%$ to $0.06 \%$, and the minimum detectable absorption per unit path length, $\kappa_{\min }$, would be reduced by a factor of nearly 50 .

Considering the path length per pass, Equation (5) implies that increasing the path length should improve the detection sensitivity (i.e. reduce the detection limit). This is certainly true for gas-phase measurements, but unfortunately is not always the case for liquid-phase measurements. Increasing the path length per pass often leads to a signficant increase in scattering and absorption losses associated with the solvent, and also increases any uncertainties associated with blank measurements where these are critical to the measurement. The effect of path length on detection sensitivity is best investigated on a case by case basis depending on the particular application at hand.

Improving the reliability of the ringdown measurement itself is perhaps best achieved by moving from a pulsed laser source to a continuous wave (CW) source. This was demonstrated convincingly by Zare and coworkers in their experiments employing the custom Brewster cell described above. Replacing the pulsed light source employed in their earlier experiments [27] with a single-mode, $\mathrm{CW}$ source allowed excitation of a single cavity mode and reduced the shot-to-shot variation in the measured value of the ringdown time from $1 \%$ to $0.04 \%$, reducing the associated experimental uncertainty accordingly.

In summary, we have seen that cavity-enhanced spectroscopies employing a standard two-mirror optical cavity provide a fairly general detection method for microfluidics and other small-volume liquid-phase sensing applications, and there is considerable scope for developing a commercial instrument with these capabilities. The requirements for developing compact stand-alone sensors employing cavity- 
enhanced detection techniques are more demanding, and will require the development of high-finesse miniature cavities suitable for integration into microfluidic channels. There are a number of candidate technologies for fabricating microcavities, with perhaps one of the most interesting being the recently-reported femtolitre tunable optical cavity arrays developed by Dolan et al [166]. Such sensors could potentially be developed for a broad range of applications spanning the chemical, biological, environmental, forensic and medical sciences.

\section{References}

1. M. Trojanowicz, Anal. Chim. Acta 653, 36 (2009)

2. P.S. Dittrich, A. Manz, Anal. Bioanal. Chem. 382, 1771 (2005)

3. S.A. Leung, R.F. Winkle, R.C.R. Wootton, A.J. deMello, Analyst 130, 46 (2005)

4. G. Cristobal, L. Arbouet, F. Sarrazin, D. Talaga, J.L. Bruneel, M. Joanicot, L. Servant, Lab Chip 6, 1140 (2006)

5. C. Massin, F. Vincent, A. Homsy, K. Ehrmann, G. Boero, P.A. Besse, A. Daridon, E. Verpoorte, N.F. de Rooij, R.S. Popovic, J. Magn. Reson., Ser A 164(2), 242 (2003)

6. S. Koster, E. Verpoorte, Lab Chip 7, 1394 (2007)

7. C. Yi, Q. Zhang, C.W. Li, J. Yang, J. Zhao, M. Yang, Anal. Bioanal. Chem. 384(6), 1259 (2006)

8. B. Kuswandi, Nuriman, J. Huskens, W. Verboom, Anal. Chim. Acta 601, 141 (2007)

9. K.B. Mogensen, J.P. Kutter, Electrophoresis 30, S92 (2009)

10. M.A. Schwarz, P.C. Hauser, Lab Chip 1, 1 (2001)

11. K.B. Mogensen, H. Klank, J.P. Kutter, Electrophoresis 25, 3498 (2004)

12. A.J. deMello, Nature 442, 394 (2006)

13. F.B. Myers, L.P. Lee, Lab Chip 8, 2015 (2008)

14. M. O’Toole, D. Diamond, Sensors 8, 2453 (2008)

15. J.C. Jokerst, J.M. Emory, C.S. Henry, Analyst 137(1), 24 (2012)

16. G. Berden, R. Peeters, G. Meijer, Int. Rev. Phys. Chem. 19(4), 565 (2000)

17. F.M. Schmidt, A. Foltynowicz, W. Ma, T. Lock, A. O., Opt. Express 15(7), 10822 (2007)

18. A. O'Keefe, D.A.G. Deacon, Rev. Sci. Instrum. 59, 2544 (1988)

19. M.D. Wheeler, S.M. Newman, A.J. Orr-Ewing, M.N.R. Ashfold, Journal of the Chemical Society-Faraday Transactions 94(3), 337 (1998)

20. J.J. Scherer, J.B. Paul, A. O’Keefe, R.J. Saykally, Chem. Rev. 97, 25 (1997)

21. S.M. Ball, R.L. Jones, Chemical Reviews 103(12), 5239 (2003)

22. B. Cummings, M.L. Hamilton, L. Ciaffoni, T.R. Pragnell, R. Peverall, G.A.D. Ritchie, G. Hancock, P.A. Robbins, J. Appl. Physiol. 111(1), 303 (2011)

23. M.R. McCurdy, Y. Bakhirkin, G. Wysocki, R. Lewicki, F.K. Tittel, J. Breath Res. 1, 014001/1 (2007)

24. G. Neri, A. Lacquaniti, G. Rizzo, N. Donato, M. Latino, M. Buemi, Nephrology Dialysis Transplantation 27(7), 2945 (2012)

25. C. Wang, Sensors 9, 7595 (2009)

26. J. Wojitas, Z. Bielecki, T. Stacewicz, J. Mikolajczyk, M. Nowakowski, Optoelectronics Review 20(1), 26 (2012)

27. K.L. Snyder, R.N. Zare, Anal. Chem. 75(13), 3086 (2003)

28. S.C. Xu, G.H. Sha, J.C. Xie, Rev. Sci. Instrum. 73(2), 255 (2002)

29. L. van der Sneppen, F. Ariese, C. Gooijer, W. Ubachs, J. Chromatogr. A 1148(2), 184 (2007)

30. K.L. Bechtel, R.N. Zare, A.A. Kachanov, S.S. Sanders, B.A. Paldus, Anal. Chem. 77(4), 1177 (2005)

31. A.J. Alexander, Anal. Chem. 78(15), 5597 (2006) 
32. S.E. Fiedler, A. Hese, A.A. Ruth, Rev. Sci. Instrum. 76, 023107 (2005)

33. T. McGarvey, A. Conjusteau, H. Mabuchi, Opt. Express 14(22), 10441 (2006)

34. M. Islam, N. Seetohul, Z. Ali, Appl. Spectrosc. 61(6), 649 (2007)

35. N. Seetohul, Z. Ali, M. Islam, Anal. Chem. 81, 4106 (2009)

36. N. Seetohul, Z. Ali, M. Islam, Analyst 134, 1887 (2009)

37. A. O'Keefe, Chem. Phys. Lett. 293, 331 (1998)

38. R. Engeln, G. Berden, R. Peeters, G. Meijer, Rev. Sci. Instrum. 69(11), 3763 (1998)

39. P.K. Dasgupta, J.S. Rhee, Anal. Chem. 59(5), 783 (1987)

40. B. Bakowski, L. Corner, G. Hancock, R. Kotchie, R. Peverall, G.A.D. Ritchie, Appl. Opt. 75(6-7), 745 (2002)

41. S.E. Fiedler, A. Hese, A.A. Ruth, Chem. Phys. Lett. 371(3-4), 284 (2003)

42. IUPAC compendium of chemical terminology. http://goldbook.iupac.org

43. M. Mazurenka, A.J. Orr-Ewing, R. Peverall, G.A.D. Ritchie, Ann. Rep. Prog. Chem., Sect. C 101, 100 (2005)

44. C.M. Rushworth, J. Davies, J.T. Cabral, P.R. Dolan, J.M. Smith, C. Vallance, Chem. Phys. Lett. (In press 2012)

45. A.C.R. Pipino, J.W. Hudgens, R.E. Huie, Chem. Phys. Lett. 280(1-2), 104 (1997)

46. A.M. Shaw, T.E. Hannon, F.P. Li, R.N. Zare, J. Phys. Chem. B 107(29), 7070 (2003)

47. J.R. O'Reilly, C.P. Butts, I.A. I'Anson, A.M. Shaw, J. Am. Chem. Soc. 127(6), 1632 (2005)

48. A.C.R. Pipino, J.W. Hudgens, R.E. Huie, Rev. Sci. Instrum. 68(8), 2978 (1997)

49. A.C.R. Pipino, Phys. Rev. Lett. 83(15), 3093 (1999)

50. A.C.R. Pipino, App. Opt. 39(9), 1449 (2000)

51. T. Von Lerber, M.W. Sigrist, Appl. Opt. 41(18), 3567 (2002)

52. D.E. Vogler, M.G. Mülller, M.W. Sigrist, Appl. Opt. 42(27), 5413 (2003)

53. D.E. Vogler, A. Lorencak, J.M. Rey, M.W. Sigrist, Opt. Laser Eng. 43(3-5), 527 (2005)

54. M. Gupta, H. Jiao, A. O'Keefe, Opt. Lett. 27(21), 1878 (2002)

55. R.S. Brown, I. Kozin, Z. Tong, R.D. Oleschuk, H.P. Loock, J. Chem. Phys. 117(23), 10444 (2002)

56. H. Waechter, J. Litman, A.H. Cheung, J.A. Barnes, H.P. Loock, Sensors 10(3), 1716 (2010)

57. C. Wang, Opt. Eng. 44, 030503 (2005)

58. C. Wang, S.T. Scherrer, Appl. Opt. 43(35), 6458 (2004)

59. P.B. Tarsa, D.M. Brzozowski, P. Rabinowitz, K.K. Lehmann, Opt. Lett. 29, 1339 (2004)

60. N. Ni, C.C. Chan, L. Xia, P. Shum, IEEE Photo. Technol. Lett. 20(16), 1351 (2009)

61. R. Engeln, G. Meijer, Rev. Sci. Instrum. 67(8), 2708 (1996)

62. E. Hamers, D. Schram, R. Engeln, Chem. Phys. Lett. 365, 237243 (2002)

63. A. Czyżewski, S. Chudzyński, K. Ernst, G. Karasizński, L. Kilianek, A. Pietruczuk, W. Skubiszak, K. Stacewicz, T.and Stelmaszczyk, B. Koch, P. Rairoux, Opt. Comm. 191(3-6), 271 (2001)

64. J.J. Scherer, Chem. Phys. Lett. 292, 143 (1998)

65. J.J. Scherer, J.B. Paul, H. Jiao, A. O'Keefe, Appl. Opt. 40(36), 6725 (2001)

66. S.N. Ball, I.M. Povey, E.G. Norton, R.L. Jones, Chem. Phys. Lett. 342, 113 (2001)

67. M. Bitter, S.M. Ball, I.M. Povey, R.L. Jones, Atm. Chem. Phys. 5, 2547 (2005)

68. C. Vallance, New J. Chem. 29, 867 (2005)

69. S.E. Fiedler, G. Hoeheisel, A.A. Ruth, A. Hese, Chemical Physics Letters 382(3-4), 447 (2003)

70. A.A. Ruth, J. Orphal, S.E. Fiedler, Appl. Opt. 46, 3611 (2007)

71. D. Venables, T. Gherman, J. Orphal, Env. Sci. Tech. 40, 6758 (2006)

72. J. Orphal, A.A. Ruth, Opt. Express 16, 1595 (2008)

73. S. Vaughan, T. Gherman, A.A. Ruth, J. Orphal, Phys. Chem. Chem. Phys. 10, 4471 (2008)

74. A. Ruth, R. Varma, D. Venables, EGU General Assembly 2009 11, 12954 (2009)

75. S.M. Ball, J.M. Langridge, R.L. Jones, Chem. Phys. Lett. 398(1-3), 68 (2004)

76. J.M. Langridge, S.M. Ball, R.L. Jones, The Analyst 131, 916 (2006)

77. J.M. Langridge, S.M. Ball, A.J.L. Shillings, R.L. Jones, Rev. Sci. Instrum. 79, 123110 (2008)

78. T. Wu, W. Zhao, W. Chen, W. Zhang, X. Gao, Appl. Phys. B 94, 85 (2008)

79. T. Wu, C. W., E. Fertein, F. Cazier, D. Dewaele, X. Gao, Appl. Phys. B 106, 501 (2011) 
80. M. Triki, P. Cermak, G. Mejean, D. Romanini, Appl. Phys. B 91, 195 (2010)

81. I. Ventrillard-Courtillot, E. Sciamma O’Brien, S. Kassi, G. Mejean, D. Romanini, Appl. Phys. B 101, 661 (2010)

82. J.M. Langridge, T. Laurila, R.S. Watt, R.L. Jones, C.F. Kaminski, J. Hult, Opt. Express 16, $10178(2008)$

83. T. Watt, R. S. ad Laurila, C.F. Kaminski, J. Hult, Applied Spectroscopy 63, 1389 (2009)

84. P.S. Johnston, K.K. Lehmann, Opt. Expr. 16, 15013 (2008)

85. S.R.T. Neil, C.M. Rushworth, C. Vallance, S.R. Mackenzie, Lab Chip 11, 3953 (2011)

86. S.S. Kiwanuka, T. Laurila, C.F. Kaminski, Anal. Chem. 82, 7498 (2010)

87. M. Schnippering, P.R. Unwin, J. Hult, T. Laurila, C.F. Kaminski, J.M. Langridge, R.L. Jones, M. Mazurenka, S.R. Mackenzie, Electrochem. Comm. 10, 1827 (2008)

88. L. van der Sneppen, C. Hancock, G.and Kaminski, T. Laurila, S.R. Mackenzie, S.R.T. Neil, R. Peverall, G.A.D. Ritchie, M. Schnippering, P.R. Unwin, The Analyst 135, 133 (2010)

89. T. Gherman, S. Kassi, A. Campargue, D. Romanini, Chem. Phys. Lett. 383, 353 (2004)

90. C. Gohle, B. Stein, A. Schliesser, T. Udem, T. Hänsch, Phys. Rev. Lett. 99, 1 (2007)

91. M. Thorpe, J. Ye, Appl. Phys. B 91, 397 (2008)

92. B. Bernhardt, A. Ozawa, P. Jacquet, M. Jacquey, Nature Photonics 4, 2009 (2009)

93. K.B. Mogensen, J. El-Ali, A. Wolff, J.P. Kutter, Appl. Opt. 42(19), 4072 (2003)

94. K.B. Mogensen, F. Eriksson, O. Gustafsson, R.P.H. Nikolajsen, J.P. Kutter, Electrophoresis 25, 3788 (2004)

95. M.H. Wu, H. Cai, X. Xu, J.P.G. Urban, Z.F. Cui, Z. Cui, Biomed. Devices 7(4), 323 (2005)

96. R. Jindal, S.M. Cramer, J. Chromatogr. A 1044, 277 (2004)

97. J.P. Landers, Handbook of capillary and microchip electrophoresis and associated microtechniques (CRC Press, 2007)

98. Y. Xue, E.S. Yeung, Anal. Chem. 66, 3575 (1994)

99. R. Alves-Segundo, N. Ibañez Garcia, M. Baeza, M. Puyol, J. Alonso-Chamarro, Microchim. Acta 172(1), 225 (2011)

100. N.J. Petersen, K.B. Mogensen, J.P. Kutter, Electrophoresis 23, 3528 (2002)

101. S.E. Moring, R.T. Reel, R.E.J. van Soest, Anal. Chem. 65(23), 3454 (1993)

102. K.W. Ro, K. Lim, B.C. Shim, J.H. Hahn, Anal. Chem. 77(16), 5160 (2005)

103. T.M. Tiggelaar, T.T. Veenstra, R.G.P. Sanders, J.G.E. Gardeniers, M.C. Elwenspoek, A. van der Berg, Talanta 56, 331 (2002)

104. J. Steigert, M. Grumann, M. Dube, W. Streule, L. Riegger, T. Brenner, P. Koltay, K. Mittmann, R. Zengerle, J. Ducrée, Sens. Actuators, A 130-131, 228 (2006)

105. T. Wang, J.H. Aiken, C.W. Huie, R.A. Hartwick, Anal. Chem. 63(14), 1372 (1991)

106. H. Salimi-Moosavi, Y. Jiang, L. Lester, G. McKinnon, D.J. Harrison, Electrophoresis 21, $1291(2000)$

107. P.S. Ellis, A.J. Lyddy-Meaney, P.J. Worsfold, I.D. McKelvie, Anal. Chim. Acta 499(1-2), 81 (2003)

108. E. Verpoorte, A. Manz, H. Lüdi, A.E. Bruno, F. Maystre, B. Krattiger, H.M. Widmer, B.H. van der Schoot, N.F. De Rooij, Sens. Actuators, B 6, 66 (1992)

109. A. Llobera, R. Wilke, S. Büttgenbach, Lab Chip 4, 24 (2004)

110. A. Llobera, R. Wilke, S. Büttgenbach, Lab Chip 5, 506 (2005)

111. A. Llobera, S. Demming, R. Wilke, S. Büttgenbach, Lab Chip 7, 1560 (2007)

112. S. Demming, A. Llobera, R. Wilke, S. Büttgenbach, Sens. Actuators, B 139, 166 (2009)

113. J.Z. Pan, B. Yao, Q. Fang, Anal. Chem. 82(8), 3394 (2010)

114. L. Billot, A. Plecis, Y. Chen, Microelectron. Eng. 85, 1269 (2008)

115. D. Psaltis, S.R. Quake, C. Yang, Nature 442, 381 (2006)

116. B. Helbo, A. Kristensen, A. Menon, J. Micromech. Microeng. 13, 307 (2003)

117. H. Shao, D. Kumar, S.A. Feld, K.L. Lear, J. MEMS 14(4), 756 (2005)

118. M. Gersborg-Hansen, S. Balslev, N.A. Mortensen, A. Kristensen, Microelectron. Eng. 78, 185 (2005)

119. J.C. Galas, C. Peroz, Q. Kou, Y. Chen, Appl. Phys. Lett. 89, 224101 (2006)

120. D. James, B. Oag, C.M. Rushworth, J.W.L. Lee, J. Davies, J.T. Cabral, C. Vallance, RSC Advances 2, 5376 (2012) 
121. I. Shestopalov, J.D. Tice, R.F. Ismagilov, Lab Chip 4, 316 (2004)

122. B. Zheng, L.S. Roach, R.F. Ismagilov, J. Am. Chem. Soc. 125(37), 11170 (2003)

123. H. Song, J.D. Tice, R.F. Ismagilov, Angew. Chem. Int. Ed. 42(7), 767 (2003)

124. H. Song, R.F. Ismagilov, J. Am. Chem. Soc. 125(47), 14613 (2003)

125. Z.T. Cygan, J.T. Cabral, K.L. Beers, E.J. Amis, Langmuir 21(8), 3629 (2005)

126. H. Song, D.L. Chen, R.F. Ismagilov, Angew. Chem. Int. Ed. 45(44), 7336 (2006)

127. S. Rudic, R.E.H. Miles, A.J. Orr-Ewing, J.P. Reid, Appl. Opt. 46, 6142 (2007)

128. R.E.H. Miles, S. Rudic, A.J. Orr-Ewing, J.P. Reid, Physical Chemistry Chemical Physics 12(15), 3914 (2010)

129. R. Engeln, G. von Helden, G. Berden, G. Meijer, Chem. Phys. Lett. 262, 105 (1996)

130. Z. Tong, A. Wright, T. McCormick, R. Li, R.D. Oleschuk, H.P. Loock, Anal. Chem. 76(22), 6594 (2004)

131. R. Li, H.P. Loock, R.D. Oleschuk, Anal. Chem. 78(16), 5685 (2006)

132. H. Waechter, K. Bescherer, C.J. Dürr, R.D. Oleschuk, H.P. Loock, Anal. Chem. 81, 9048 (2009)

133. H. Waechter, D. Munzke, A. Jang, H.P. Loock, Anal. Chem. 83, 2719 (2011)

134. P.B. Tarsa, P. Rabinowitz, K.K. Lehmann, Chem. Phys. Lett. 383(3-4), 297 (2004)

135. C.M. Rushworth, D. James, C.J.V. Jones, C. Vallance, Opt. Lett. 36(15), 2952 (2011)

136. C.M. Rushworth, D. James, J.W.L. Lee, C. Vallance, Anal. Chem. 83, 8492 (2011)

137. Z. Tong, M. Jakubinek, A. Wright, A. Gillies, H.P. Loock, Rev. Sci. Instrum. 74(11), 4818 (2003)

138. M. Andachi, T. Nakayama, M. Kawasaki, S. Kurokawa, H.P. Loock, Appl. Phys. B 88, 131 (2007)

139. G. Stewart, K. Atherton, H. Yu, B. Culshaw, Meas. Sci. Technol. 12, 843 (2001)

140. G. Stewart, K. Atherton, B. Culshaw, Opt. Lett. 29(5), 442 (2004)

141. URL http://www.argo.ucsd.edu/

142. B.P. W., T. Jickells, C.S. Law, S. Blain, E.A. Boyle, K.O. Buesseler, K.H. Coale, J.J. Cullen, H.J.W. de Baar, M. Follows, M. Harvey, C. Lancelot, M. Levasseur, N.P.J. Owens, R. Pollard, R.B. Rivkin, J. Sarmiento, V. Schoemann, V. Smetacek, S. Takeda, A. Tsuda, S. Turner, A.J. Watson, Science 315, 612 (2007)

143. J. Moorthy, G.A. Mensing, D. Kim, S. Mohanty, D.T. Eddington, W.H. Tepp, E.A. Johnson, D.J. Beebe, Electrophoresis 25, 1705 (2004)

144. V. Srinivasan, V.K. Pamula, R.B. Fair, Anal. Chim. Acta 507, 145 (2004)

145. M. Grumann, J. Steigert, L. Riegger, I. Moser, B. Enderle, K. Riebeseel, G. Urban, R. Zengerle, J. Ducrée, Biomed. Microdevices 8, 209 (2006)

146. K. Grasshoff, K. Kremling, M. Ehrhardt, Methods of Seawater Analysis, 3rd edn. (Wiley$\mathrm{VCH}, 2007)$

147. F.J. Millero, Chemical Oceanography, 3rd edn. (CRC Press, 2005)

148. WHO, Guidelines for drinking-water quality, 4 th edn. (World Health Organization, Geneva, Switzerland, 2011)

149. D.R. Turner, K.A. Hunter, The biogeochemistry of iron in seawater (John Wiley and Sons, 2001)

150. P. Griess, Ber. dt. chem. Ges. 12, 426 (1879)

151. D.F. Boltz, J.A. Howell, Colorimetric Determination of Nonmetals (John Wiley and Sons Inc., 1978)

152. J.V. Sieben, C.F.A. Floquet, I.R.G. Ogilvie, M.C. Mowlem, H. Morgan, Anal. Methods 2, $484(2010)$

153. E.B. Sandell, Colorimetric determination of traces of metals (John Wiley and Sons Inc., 1959)

154. E.P. Achterberg, T.W. Holland, A.R. Bowie, R.F.C. Mantoura, P.J. Worsfold, Anal. Chim. Acta 442, 1 (2001)

155. S. Pehkonen, Analyst 120, 2655 (1995)

156. G.F. Smith, W.H. McCurdy, H. Diehl, Analyst 77, 418 (1952)

157. D.W. King, J. Lin, D.R. Kester, Anal. Chim. Acta 247, 125 (1991) 
158. P. Raimbault, G. Slawyk, B. Coste, J. Fry, Mar. Biol. 104(2), 347 (1990)

159. C.F.A. Floquet, V.J. Sieben, A. Milani, E.P. Joly, I.R.G. Ogilvie, H. Morgan, M. Mowlem, Talanta 84, 235 (2011)

160. E.T. Steimle, E.A. Kaltenbacher, R.H. Byrne, Mar. Chem. 77, 255 (2002)

161. R.D. Waterbury, W. Yao, R.H. Byrne, Anal. Chim. Acta 357(12), 99 (1997)

162. J.Z. Zhang, Deep Sea Res. Part I 47(6), 1157 (2000)

163. J.Z. Zhang, C. Kelbe, F.J. Millero, Anal. Chim. Acta 438, 49 (2001)

164. S. Blain, P. Tréguer, Anal. Chim. Acta 308, 425 (1995)

165. J.L. Manzoori, S. Soflaee, Anal. Lett. 34(2), 231 (2001)

166. P.R. Dolan, G.M. Hughes, F. Grazioso, B.R. Patton, J.M. Smith, Opt. Lett. 35(21), 3556 (2010) 